\title{
Swallowtail Band Structure of the Superfluid Fermi Gas in an Optical Lattice
}

\author{
Gentaro Watanabe, ${ }^{1,2,3}$ Sukjin Yoon, ${ }^{1}$ and Franco Dalfovo ${ }^{4}$ \\ ${ }^{1}$ Asia Pacific Center for Theoretical Physics (APCTP), Pohang, Gyeongbuk 790-784, Korea \\ ${ }^{2}$ Department of Physics, POSTECH, Pohang, Gyeongbuk 790-784, Korea \\ ${ }^{3}$ Nishina Center, RIKEN, 2-1 Hirosawa, Wako, Saitama 351-0198, Japan \\ ${ }^{4}$ INO-CNR BEC Center and Department of Physics, University of Trento, 38123 Povo, Italy
}

(Dated: August 6, 2018)

\begin{abstract}
We investigate the energy band structure of the superfluid flow of ultracold dilute Fermi gases in a one-dimensional optical lattice along the BCS to BEC crossover within a mean-field approach. In each side of the crossover region, a loop structure (swallowtail) appears in the Bloch energy band of the superfluid above a critical value of the interaction strength. The width of the swallowtail is largest near unitarity. Across the critical value of the interaction strength, the profiles of density and pairing field change more drastically in the BCS side than in the BEC side. It is found that along with the appearance of the swallowtail, there exists a narrow band in the quasiparticle energy spectrum close to the chemical potential and the incompressibility of the Fermi gas consequently experiences a profound dip in the BCS side, unlike in the BEC side.
\end{abstract}

PACS numbers: 03.75.Ss, 67.85.De, 67.85.Hj, 03.75.Lm

Ultracold atoms in optical lattices attract much interest because the controllability of both the lattice geometry and the interatomic interaction is such that they serve as testing beds for various models [1]. For Bose-Einstein condensates (BECs), it has been pointed out that the interaction can change the Bloch band structure drastically, causing the appearance of a loop structure called "swallowtail" in the energy dispersion $[2,3]$. This is due to the competition between the external periodic potential and the nonlinear mean-field interaction: the former favors a sinusoidal band structure, while the latter tends to make the density smoother and the energy dispersion quadratic. When the nonlinearity wins, the effect of the external potential is screened and a swallowtail energy loop appears [4]. This nonlinear effect requires the existence of an order parameter and, consequently, the emergence of swallowtails can be viewed as a peculiar manifestation of superfluidity in periodic potentials.

The problem of swallowtails can be even more important in Fermi superfluids due to the possible wide implications for various systems in condensed matter physics and nuclear physics. Indeed, extensive recent research activities are devoted to the simulation of solid states using cold Fermi gases and the behavior of cold fermions in optical lattices can lead to interesting analogies with superconductors and superconductor superlattices. In addition, our work may have implications also for superfluid neutrons in neutron stars, especially those in "pasta" phases (see, e.g., Ref. [5] and references therein) in neutron star crusts [6], where nuclei form a crystalline lattice in which superfluid neutrons can flow. However, unlike the Bose case [2-4, 7-9], little has been studied in this problem so far and a fundamental question of whether or not swallowtails exist along the crossover from the Bardeen-Cooper-Schrieffer (BCS) to BEC states is still open. In this context, our work is aimed at showing the existence and the conditions for emergence of swallowtails in Fermi superfluids and presenting the unique features which make them different from those in bosons.

We consider a two-component unpolarized dilute Fermi gas made of atoms of mass $m$ interacting with $s$-wave scattering length $a_{s}$ and subject to a one-dimensional (1D) optical lattice of the form $V_{\text {ext }}(\mathbf{r})=s E_{\mathrm{R}} \sin ^{2} q_{\mathrm{B}} z \equiv$ $V_{0} \sin ^{2} q_{\mathrm{B}} z$. Here, $V_{0} \equiv s E_{\mathrm{R}}$ is the lattice height, $s$ is the lattice intensity in dimensionless units, $E_{\mathrm{R}}=\hbar^{2} q_{\mathrm{B}}^{2} / 2 m$ is the recoil energy, $q_{\mathrm{B}}=\pi / d$ is the Bragg wave vector and $d$ is the lattice constant. We compute the energy band structure of the system by solving the Bogoliubovde Gennes (BdG) equations [10]:

$$
\left(\begin{array}{cc}
H(\mathbf{r}) & \Delta(\mathbf{r}) \\
\Delta^{*}(\mathbf{r}) & -H(\mathbf{r})
\end{array}\right)\left(\begin{array}{c}
u_{i}(\mathbf{r}) \\
v_{i}(\mathbf{r})
\end{array}\right)=\epsilon_{i}\left(\begin{array}{c}
u_{i}(\mathbf{r}) \\
v_{i}(\mathbf{r})
\end{array}\right)
$$

where $H(\mathbf{r})=-\hbar^{2} \nabla^{2} / 2 m+V_{\text {ext }}(\mathbf{r})-\mu, u_{i}(\mathbf{r})$ and $v_{i}(\mathbf{r})$ are quasiparticle wavefunctions, and $\epsilon_{i}$ is the corresponding quasiparticle energy. The chemical potential $\mu$ is determined from the constraint on the particle number $N=2 \sum_{i} \int\left|v_{i}(\mathbf{r})\right|^{2} d \mathbf{r}$ and the pairing field $\Delta(\mathbf{r})$ should satisfy a self-consistency condition $\Delta(\mathbf{r})=$ $-g \sum_{i} u_{i}(\mathbf{r}) v_{i}^{*}(\mathbf{r})$, where $g$ is the coupling constant for the $s$-wave contact interaction which needs to be renormalized. In the presence of a supercurrent with wavevector $Q=P / \hbar\left(|P| \leq P_{\text {edge }} \equiv \hbar q_{\mathrm{B}} / 2\right)$ moving in the $z$-direction [11], one can write the quasiparticle wavefunctions in the Bloch form as $u_{i}(\mathbf{r})=\tilde{u}_{i}(z) e^{i Q z} e^{i \mathbf{k} \cdot \mathbf{r}}$ and $v_{i}(\mathbf{r})=\tilde{v}_{i}(z) e^{-i Q z} e^{i \mathbf{k} \cdot \mathbf{r}}$ leading to the pairing field as $\Delta(\mathbf{r})=e^{i 2 Q z} \tilde{\Delta}(z)$. Here $\tilde{\Delta}(z), \tilde{u}_{i}(z)$, and $\tilde{v}_{i}(z)$ are complex functions with period $d$ and the wave vector $k_{z}$ $\left(\left|k_{z}\right| \leq q_{\mathrm{B}}\right)$ lies in the first Brillouin zone. This Bloch decomposition transforms Eq. (1) into the following BdG equations for $\tilde{u}_{i}(z)$ and $\tilde{v}_{i}(z)$ :

$$
\left(\begin{array}{cc}
\tilde{H}_{Q}(z) & \tilde{\Delta}(z) \\
\tilde{\Delta}^{*}(z) & -\tilde{H}_{-Q}(z)
\end{array}\right)\left(\begin{array}{c}
\tilde{u}_{i}(z) \\
\tilde{v}_{i}(z)
\end{array}\right)=\epsilon_{i}\left(\begin{array}{c}
\tilde{u}_{i}(z) \\
\tilde{v}_{i}(z)
\end{array}\right),
$$



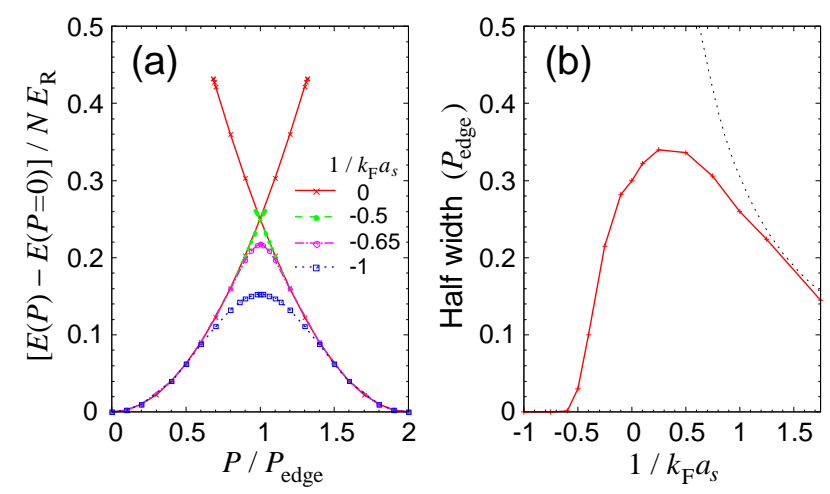

FIG. 1: (Color online) (a) Energy $E$ per particle as a function of the quasimomentum $P$ for various values of $1 / k_{\mathrm{F}} a_{s}$; (b) half-width of the swallowtails along the BCS-BEC crossover. These results are obtained for $s=0.1$ and $E_{\mathrm{F}} / E_{\mathrm{R}}=2.5$. The quasimomentum $P_{\text {edge }}=\hbar q_{\mathrm{B}} / 2$ fixes the edge of the first Brillouin zone. The dotted line in (b) is the half-width in a BEC obtained by solving the GP equation; it vanishes at $1 / k_{\mathrm{F}} a_{s} \simeq 10.6$.

where

$\tilde{H}_{Q}(z) \equiv \frac{\hbar^{2}}{2 m}\left[k_{\perp}^{2}+\left(-i \partial_{z}+Q+k_{z}\right)^{2}\right]+V_{\text {ext }}(z)-\mu$.

Here, $k_{\perp}^{2} \equiv k_{x}^{2}+k_{y}^{2}$ and the label $i$ represents the wave vector $\mathbf{k}$ as well as the band index.

In the following, we mainly present the result for $s=$ $V_{0} / E_{\mathrm{R}}=0.1$ and $E_{\mathrm{F}} / E_{\mathrm{R}}=2.5$ as an example, where $E_{\mathrm{F}}=\hbar^{2} k_{\mathrm{F}}^{2} /(2 m)$ and $k_{\mathrm{F}}=\left(3 \pi^{2} n_{0}\right)^{1 / 3}$ are the Fermi energy and momentum of a uniform free Fermi gas of density $n_{0}$. These values fall in the range of parameters of feasible experiments [12].

We first compute the energy per particle in the lowest Bloch band as a function of the quasimomentum $P$ for various values of $1 / k_{\mathrm{F}} a_{s}$. The results in Fig. 1(a) show that the swallowtails appear above a critical value of $1 / k_{\mathrm{F}} a_{s}$ where the interaction energy is strong enough to dominate the lattice potential. In Fig. 1(b), the halfwidth of the swallowtails from the BCS to the BEC side is shown. It reaches a maximum near unitarity $\left(1 / k_{\mathrm{F}} a_{s}=\right.$ $0)$. In the far BCS and BEC limits, the width vanishes because the system is very weakly interacting and the band structure tends to be sinusoidal. When approaching unitarity from either side, the interaction energy increases and can dominate over the periodic potential, which means that the system behaves more like a translationally invariant superfluid and the band structure follows a quadratic dispersion terminating at a maximum $P$ larger than $P_{\text {edge }}$. In the BEC side, we compare the results of our BdG calculations with those of the GrossPitaveskii (GP) equation for bosons of mass $m_{b}=2 m$ interacting with scattering length $a_{b}=2 a_{s}[13]$ in an optical lattice $2 V_{\text {ext }}(z):-\left(\hbar^{2} / 4 m\right) \nabla^{2} \Phi(\mathbf{r})+2 V_{\text {ext }}(z) \Phi(\mathbf{r})+$ $\left(8 \pi \hbar^{2} a_{s} / 2 m\right)|\Phi(\mathbf{r})|^{2} \Phi(\mathbf{r})=\mu_{B} \Phi(\mathbf{r})$, where $\Phi(\mathbf{r})$ is a single macroscopic wavefunction describing the BEC. As it

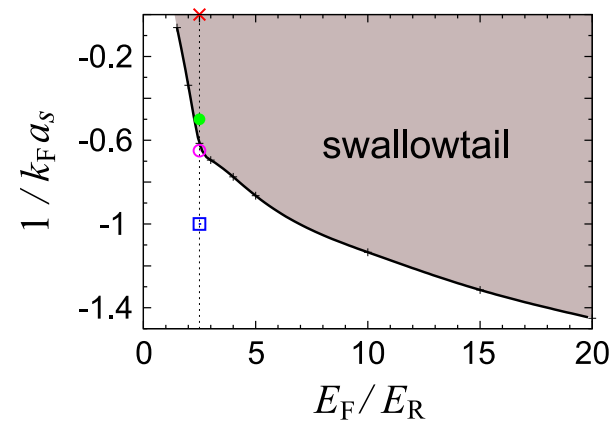

FIG. 2: (Color online) The parameter region $\left(E_{\mathrm{F}} / E_{\mathrm{R}}\right.$, $\left.1 / k_{\mathrm{F}} a_{s}\right)$ where the swallowtails appear for $s=0.1$. Symbols on the vertical line at $E_{\mathrm{F}} / E_{\mathrm{R}}=2.5$ correspond to the cases shown in Fig. 1(a).

should be, the difference between the two curves becomes vanishingly small in the deep BEC regime. With our set of parameters $\left(s=0.1\right.$ and $\left.E_{\mathrm{F}} / E_{\mathrm{R}}=2.5\right)$, the width of the swallowtail predicted by the GP equation vanishes at $1 / k_{\mathrm{F}} a_{s} \simeq 10.6$.

Whether the swallowtail appears or not depends on three parameters: the interaction parameter $1 / k_{\mathrm{F}} a_{s}$, the lattice intensity $s$, and the ratio between the Fermi energy and the recoil energy $E_{\mathrm{F}} / E_{\mathrm{R}}$. In Fig. 2, we fix $s=0.1$ and show the parameter region $\left(E_{\mathrm{F}} / E_{\mathrm{R}}\right.$, $\left.1 / k_{\mathrm{F}} a_{s}\right)$ where we find swallowtails in the BCS side of the crossover $\left(1 / k_{\mathrm{F}} a_{s}<0\right)[14]$. We see that for weaker interaction (i.e., larger values of $1 / k_{\mathrm{F}}\left|a_{s}\right|$ ) higher densities (larger values of $E_{\mathrm{F}}$ ) are required to create swallowtails, as expected. The $s$-dependence of critical values of $1 / k_{\mathrm{F}} a_{s}$ in the BCS side is much weaker than the $1 / s$ scaling behavior in the BEC side. In the BCS side, as far as we have checked in $0.1 \leq s \leq 0.5$, the critical value of $1 / k_{\mathrm{F}} a_{s}$ changes within $30 \%$ at $E_{\mathrm{F}} / E_{\mathrm{R}}=5$ and the change gets smaller with increasing $E_{\mathrm{F}} / E_{\mathrm{R}}$. This weak dependence of the swallowtail region on $s$ in the BCS side is due to the Fermi statistics: Provided $E_{\mathrm{F}} / V_{0}=\left(E_{\mathrm{F}} / E_{\mathrm{R}}\right) / s$ is sufficiently larger than unity, the flow of the BCS condensate formed from fermions near the Fermi surface is not very sensitive to the presence of the lattice potential, while the flow of the BEC formed from bosonic dimers all at the bottom of the energy levels is more sensitive to it.

Both the pairing field and the density exhibit interesting features in the range of parameters where the swallowtails appear. This is particularly evident at the Brillouin zone boundary, $P=P_{\text {edge }}$. In Fig. 3 , we show the magnitude of the pairing field $|\Delta(z)|$ and the density $n(z)$ calculated at the minimum $(z=0)$ and at the maximum $(z= \pm d / 2)$ of the lattice potential. In general, $n(z)$ and $|\Delta(z)|$ take maximum (minimum) values where the external potential takes its minimum (maximum) values (for the full profiles, see Supplemental Material [15]). The figure shows that $|\Delta(d / 2)|$ remains zero in the BCS regime 

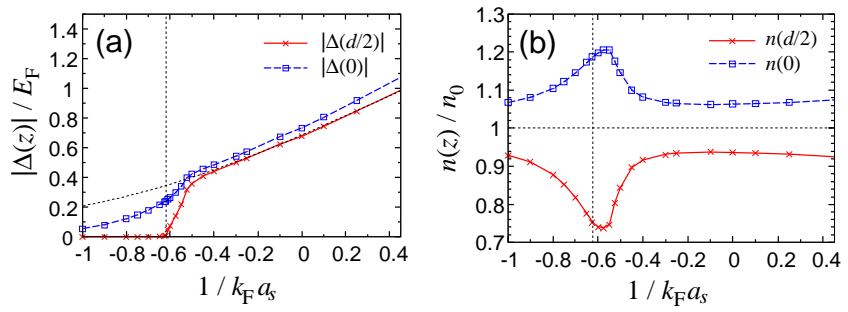

FIG. 3: (Color online) Profiles of (a) the pairing field $|\Delta(z)|$ and (b) the density $n(z)$ along the change of $1 / k_{\mathrm{F}} a_{s}$ for $P=$ $P_{\text {edge }}$ in the case of $s=0.1$ and $E_{\mathrm{F}} / E_{\mathrm{R}}=2.5$. The values of $|\Delta(z)|$ and $n(z)$ at the minimum $(z=0$, blue $\square)$ and at the maximum $(z= \pm d / 2$, red $\times)$ of the lattice potential are shown. The vertical dotted lines show the critical value of $1 / k_{\mathrm{F}} a_{s}$ above which the swallowtail exists. The dotted curve in (a) shows $|\Delta|$ in the uniform system.

until the swallowtail appears at $1 / k_{\mathrm{F}} a_{s} \approx-0.62$. Then it increases abruptly to values comparable to $|\Delta(0)|$, which means that the pairing field becomes almost uniform at $P=P_{\text {edge }}$ in the presence of swallowtails. As regards the density, we find that the amplitude of the density variation, $n(0)-n(d / 2)$, exhibits a pronounced maximum near the critical value of $1 / k_{\mathrm{F}} a_{s}$. In contrast, in the BEC side, the order parameter and the density are smooth monotonic functions of the interaction strength even in the region where the swallowtail appears. At $P=P_{\text {edge }}$, the solution of the GP equation for bosonic dimers gives the densities $n_{b}(0)=n_{b 0}\left(1+V_{0} / 2 n_{b 0} U_{0}\right)$ and $n_{b}(d / 2)=n_{b 0}\left(1-V_{0} / 2 n_{b 0} U_{0}\right)$ with $V_{0} / 2 n_{b 0} U_{0}=$ $(3 \pi / 4)\left(s E_{\mathrm{R}} / E_{\mathrm{F}}\right)\left(1 / k_{\mathrm{F}} a_{s}\right)$, where $n_{b 0}$ is the average density of bosons and $U_{0}=4 \pi \hbar^{2} a_{b} / m_{b}[2,16,17]$. Near the critical value of $1 / k_{\mathrm{F}} a_{s}$, unlike the BCS side, the nonuniformity just decreases all the way even after the swallowtail appears. The local density at $z=d / 2$ is zero until the swallowtail appears in the BEC side while it is nonzero in the BCS side irrespective of the existence of the swallowtail. The qualitative behavior of $|\Delta(z)|$ around the critical point of $1 / k_{\mathrm{F}} a_{s}$ is similar to that of $n_{b}(z)$ because $n_{b}(\mathbf{r})=\left(m^{2} a_{s} / 8 \pi\right)|\Delta(\mathbf{r})|^{2}[18]$ in the BEC limit.

The quasiparticle energy spectrum plays an important role in determining the properties of the Fermi gas. Here we show that the emergence of swallowtails in the BCS side and for $E_{\mathrm{F}} / E_{\mathrm{R}} \gtrsim 1$ is associated with peculiar structures of the quasiparticle energy spectrum around the chemical potential. In the presence of a superflow moving in the $z$ direction with wavevector $Q$, the quasiparticle energies are given by the eigenvalues in Eq. (2). Since the potential is shallow $(s \ll 1)$, some qualitative results can be obtained even ignoring $V_{\text {ext }}(z)$ except for its periodicity. With this assumption we obtain

$$
\epsilon_{\mathbf{k}} \approx \frac{\left(k_{z}+2 q_{\mathrm{B}} l\right) Q}{m}+\sqrt{\left[\frac{k_{\perp}^{2}+\left(k_{z}+2 q_{\mathrm{B}} l\right)^{2}+Q^{2}}{2 m}-\mu\right]^{2}+|\Delta|^{2}}
$$
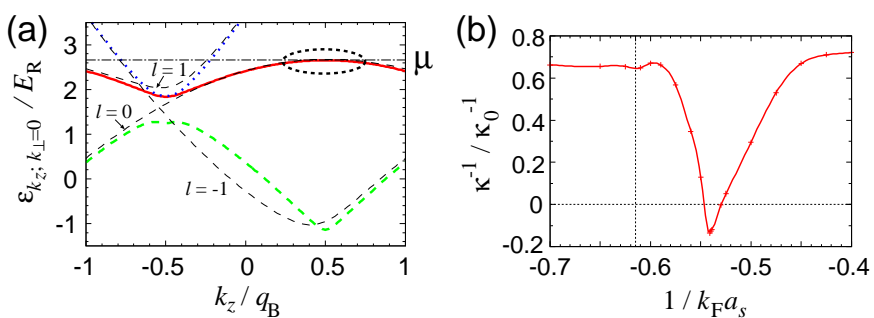

FIG. 4: (Color online) (a) Lowest three Bloch bands of the quasiparticle energy spectrum at $k_{\perp}=0$ for $P=P_{\text {edge }}$ and $1 / k_{\mathrm{F}} a_{s}=-0.62$. Thin black dashed lines labeled by l's show the approximate energy bands obtained from Eq. (3) using $\mu \simeq 2.66 E_{\mathrm{R}}$ and $|\Delta| \simeq|\Delta(0)| \simeq 0.54 E_{\mathrm{R}}$. (b) Incompressibility $\kappa^{-1}$ at $P=P_{\text {edge }}$ around the critical value of $1 / k_{\mathrm{F}} a_{s} \approx-0.62$ where the swallowtail starts to appear. The quantity $\kappa_{0}^{-1}$ is the incompressibility of the homogeneous free Fermi gas of the same average density. In both panels, we have used the values $s=0.1$ and $E_{\mathrm{F}} / E_{\mathrm{R}}=2.5$.

with $l$ being integers for the band index. If $Q=$ 0, the $l=0$ band has the energy spectrum $\sqrt{\left[\left(k_{\perp}^{2}+k_{z}^{2}\right) / 2 m-\mu\right]^{2}+|\Delta|^{2}}$ which has a local maximum at $k_{z}=k_{\perp}=0$. When $Q=P_{\text {edge }} / \hbar$, the spectrum is tilted and the local maximum moves to $k_{z} \simeq q_{\mathrm{B}} / 2$ provided $|\Delta| \ll E_{\mathrm{F}}$ (and $E_{\mathrm{F}} / E_{\mathrm{R}} \gtrsim 1$ ). In the absence of the swallowtail, the full $\mathrm{BdG}$ calculation indeed gives a local maximum at $k_{z}=q_{\mathrm{B}} / 2$ [see Fig. $4(\mathrm{a})$, where the green dashed line in the region $-1<k_{z} / q_{\mathrm{B}}<-0.5$ and the red solid line in the region $-0.5<k_{z} / q_{\mathrm{B}}<1$ correspond to the $l=0$ band] and the quasiparticle spectrum is symmetric about this point, which reflects that the current is zero. As $E_{\mathrm{F}} / E_{\mathrm{R}}$ increases, the band becomes flatter as a function of $k_{z}$ and narrower in energy.

In Fig. 4(a), we show the quasiparticle energy spectrum at $k_{\perp}=0$ for $P=P_{\text {edge. }}$. When the swallowtail is on the edge of appearing, the top of the narrow band just touches the chemical potential $\mu$ [see the dotted ellipse in Fig. 4(a)]. Suppose $1 / k_{\mathrm{F}} a_{s}$ is slightly larger than the critical value, so that the top of the band is slightly above $\mu$. In this situation a small change of the quasimomentum $P$ causes a change of $\mu$. In fact, when $P$ is increased from $P=P_{\text {edge }}$ to larger values, the band is tilted and the top of the band moves upwards; the chemical potential $\mu$ should also increase to compensate for the loss of states available. This implies $\partial \mu / \partial P>0$. (See also Supplemental Material [15].) On the other hand, since the system is periodic, the existence of a branch of stationary states with $\partial \mu / \partial P>0$ at $P=P_{\text {edge }}$ implies the existence of another symmetric branch with $\partial \mu / \partial P<0$ at the same point, thus suggesting the occurrence of a swallowtail structure.

A direct consequence of the existence of a narrow band in the quasiparticle spectrum near the chemical potential is a strong reduction of the incompressibility $\kappa^{-1}=n \partial \mu(n) / \partial n$ close to the critical value of $1 / k_{\mathrm{F}} a_{s}$ in 
the region where swallowtails exist in the BCS side [see Fig. 4(b)]. The dip of $\kappa^{-1}$ occurs in the situation where the top of the narrow band is just above $\mu$ for $P=P_{\text {edge }}$ $\left(1 / k_{\mathrm{F}} a_{s}\right.$ is slightly above the critical value). An increase of the density $n$ has little effect on $\mu$ in this case, because the density of states is large in this range of energy and the new particles can easily adjust themselves near the top of the band by a small increase of $\mu$. This implies that $\partial \mu(n) / \partial n$ is small and the incompressibility has a pronounced dip [19]. It is worth noting that in the BEC side the appearance of the swallowtail is not associated with any significant change of incompressibility. In fact, the exact solution of the GP equation gives $\kappa^{-1}=n_{b 0} U_{0}$ near the critical conditions for the occurrence of swallowtails, being a smooth and monotonic function of the interaction strength.

Swallowtails may produce observable effects in the behavior of Bloch oscillations [3, 20]. Since Bloch oscillations have various important applications, such as precision measurements of forces [21] and controlling the motion of a wave packet [22, 23], a better understanding of swallowtails in bosonic and fermionic gases is certainly useful in these contexts. One may also exploit the tunability of the interaction and the peculiar dynamics of the superfluid in the lattice for different applications. For instance, by periodically sweeping a magnetic field across the critical region for the appearance of swallowtails, one can produce a time modulation of the shape of the lowest energy band between a sinusoidal form and a quadraticlike form. Since the absolute value of the group velocity $\partial_{P}[E(P) / N]$ of the latter is always larger than that of the former [see Fig. 1(a)], one could realize a directed motion of the gas by synchronizing the period of this modulation with the period of the Bloch oscillations (see also Supplemental Material [15]). This may be experimentally more accessible in the BCS side than in the BEC side because the critical value of $1 / k_{\mathrm{F}}\left|a_{s}\right|$ is of order 1 for a wide range of $s$ and $E_{\mathrm{F}} / E_{\mathrm{R}}$. This new method would complement other proposals for realizing directed motion of atomic wave packets in $1 \mathrm{D}$ optical lattices [23, 24].

In summary, we have predicted the existence of swallowtails in the energy band of superfluid fermions in a lattice and have pointed out some key features which make these swallowtails different from those in a BEC. The results are obtained within a range of parameters compatible with current experiments [12]. We hope our predictions stimulate experiments aimed to observe swallowtails with Fermi gases.

We acknowledge C. J. Pethick, Y. Shin, and T. Takimoto for helpful discussions. This work was supported by the Max Planck Society, the Korea Ministry of Education, Science and Technology, Gyeongsangbuk-Do, Pohang City, for the support of the JRG at APCTP, and ERC through the QGBE grant. Calculations were performed on RICC in RIKEN and Wiglaf at the University of Trento.
[1] O. Morsch and M. Oberthaler, Rev. Mod. Phys. 78, 179 (2006); M. Lewenstein et al., Adv. Phys. 56, 243 (2007); I. Bloch et al., Rev. Mod. Phys. 80, 885 (2008).

[2] B. Wu, R. B. Diener, and Q. Niu, Phys. Rev. A 65, 025601 (2002).

[3] D. Diakonov et al., Phys. Rev. A 66, 013604 (2002).

[4] E. J. Mueller, Phys. Rev. A 66, 063603 (2002).

[5] G. Watanabe et al., Phys. Rev. Lett. 103, 121101 (2009).

[6] G. Watanabe et al., Phys. Rev. A 83, 033621 (2011).

[7] M. Machholm, C. J. Pethick, and H. Smith, Phys. Rev. A 67, 053613 (2003).

[8] B. T. Seaman, L. D. Carr, and M. J. Holland, Phys. Rev. A 71, 033622 (2005); ibid. 72, 033602 (2005).

[9] I. Danshita and S. Tsuchiya, Phys. Rev. A 75, 033612 (2007).

[10] Neither the hydrodynamic theory nor the tight-binding model is appropriate since they cannot describe the swallowtails correctly along the BCS-BEC crossover: the former always yields the dispersion of the quadratic form without termination and the latter gives the sinusoidal band.

[11] Nonzero values of $P$ can be obtained, for instance, by orienting the periodic potential in the direction of the gravity field or by imposing a suitable time-dependent phase to the optical lattice.

[12] D. E. Miller et al., Phys. Rev. Lett. 99, 070402 (2007).

[13] Here we use $a_{b}=2 a_{s}$ in the GP equation for consistency with the mean-field BdG theory. The exact value in the BEC limit would be $a_{b}=0.6 a_{s}$ [D. S. Petrov et al., Phys. Rev. Lett. 93, 090404 (2004)], and the corresponding width of the swallowtail obtained in experiments would be smaller in this limit.

[14] The energetic stability of swallowtails can be addressed by considering the fermionic pair-breaking excitations and long-wavelength phonon excitations [see, e.g., G. Watanabe et al., Phys. Rev. A 80, 053602 (2009)]. From the excitation spectrum one can extract the critical quasimomentum $P_{c}$ below which the system is stable. If $P_{c}$ exceeds $P_{\text {edge }}$, there exists an energetically stable region for the swallowtail. In the case of $s=0.1$ and $E_{\mathrm{F}} / E_{\mathrm{R}}=2.5$, we find stable swallowtails in the range $-0.2 \lesssim 1 / k_{\mathrm{F}} a_{s}<0.92$. However, even outside of this region, the energetic instability is not a serious obstacle for the experimental observation since the breakdown of superfluidity due to the energetic instability requires dissipation, which is inefficient at low temperatures. As far as the dynamical instability is concerned, we expect that the range of $1 / k_{\mathrm{F}} a_{s}$, where the system is dynamically stable, is larger than that for the energetic instability as known to happen in the case of BEC.

[15] See Supplemental Material for the profiles of $|\Delta(z)|$ and $n(z)$, the dependence of the quasiparticle energy spectrum on $P$, and more discussion about the directed motion.

[16] J. C. Bronski et al., Phys. Rev. Lett. 86, 1402 (2001); J. C. Bronski et al., Phys. Rev. E 63, 036612 (2001).

[17] This result is exact only when the swallowtails exist, otherwise it is approximate, but qualitatively correct.

[18] P. Pieri and G. C. Strinati, Phys. Rev. Lett. 91, 030401 (2003).

[19] With the parameters used in Fig. 4(b), the incompress- 
ibility takes negative values in a small region around $1 / k_{\mathrm{F}} a_{s}=-0.55$, which means that the system might be dynamically unstable against long-wavelength perturbations. By choosing appropriate parameters, this region disappears.

[20] B. Wu and Q. Niu, Phys. Rev. A 61, 023402 (2000).

[21] G. Roati et al., Phys. Rev. Lett. 92, 230402 (2004); G. Ferrari et al., Phys. Rev. Lett. 97, 060402 (2006); M. Fattori et al., Phys. Rev. Lett. 100, 080405 (2008).

[22] A. Alberti et al., Nature Phys. 5, 547 (2009).

[23] E. Haller et al., Phys. Rev. Lett. 104, 200403 (2010).

[24] C. Mennerat-Robilliard et al., Phys. Rev. Lett. 82, 851 (1999); Q. Thommen, J. C. Garreau, and V. Zehnlé, Phys. Rev. A 65, 053406 (2002); M. Schiavoni et al., Phys. Rev. Lett. 90, 094101 (2003); L. Sanchez-Palencia, Phys. Rev. E 70, 011102 (2004); C. E. Creffield, Phys. Rev. Lett. 99, 110501 (2007); R. Gommers et al., Phys. Rev. Lett. 100, 040603 (2008); K. Kudo and T. S. Monteiro, Phys. Rev. A 83, 053627 (2011); C. E. Creffield and F. Sols, Phys. Rev. A 84, 023630 (2011); F. Zhan et al., Phys. Rev. A 84, 043617 (2011). 


\section{Supplemental Material for Swallowtail Band Structure of the Superfluid Fermi Gas in an Optical Lattice}

Gentaro Watanabe, Sukjin Yoon, and Franco Dalfovo

Full profiles of the pairing field $|\Delta(z)|$ and the density $n(z)$

In Fig. 3 of the paper, the values of $|\Delta(z)|$ and $n(z)$ at the minimum and at the maximum of the lattice potential are shown. The full profiles of $|\Delta(z)|$ and $n(z)$ along the lattice vector ( $z$-direction) are given in the following Fig. 5. By increasing the interaction parameter $1 / k_{\mathrm{F}} a_{s}$, we find that the order parameter $|\Delta|$ at the maximum $(z= \pm d / 2)$ of the lattice potential exhibits a transition from zero to nonzero values at the critical value of $1 / k_{\mathrm{F}} a_{s}$ at which the swallowtail appears [see also Fig. 3(a) of the paper]. Note that here we plot the absolute value of $\Delta$; the order parameter $\Delta$ behaves smoothly and changes sign across zero.
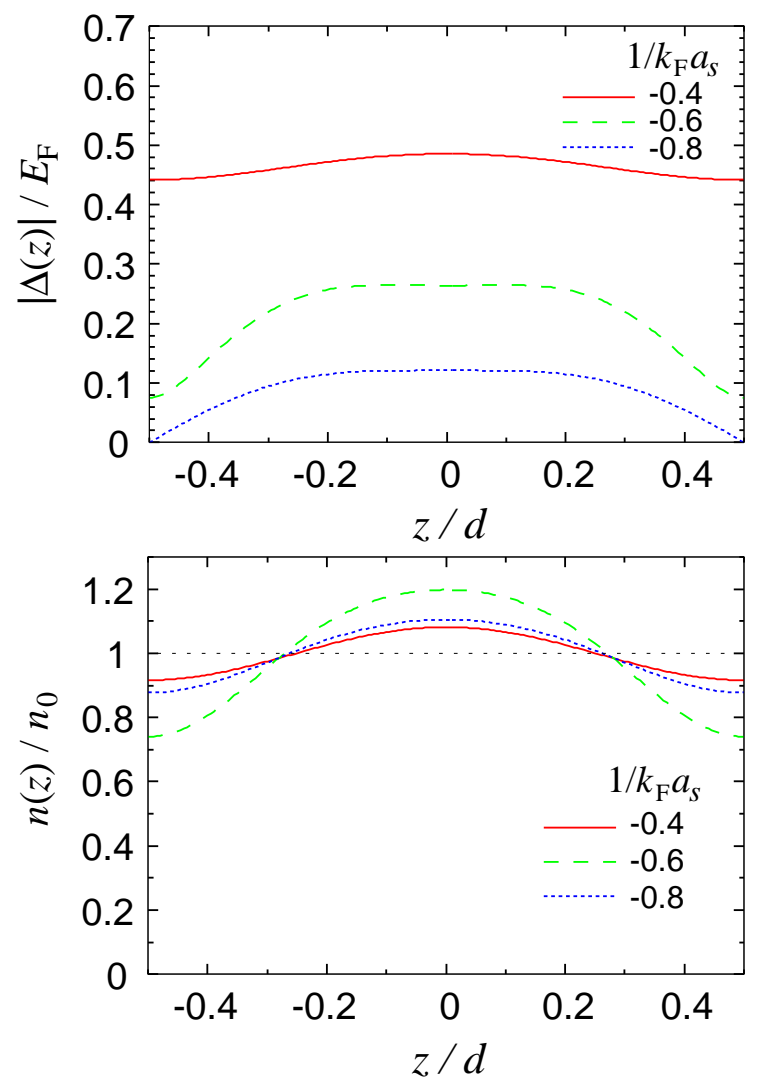

FIG. 5: Profiles of the pairing field $|\Delta(z)|$ and the density $n(z)$ at $1 / k_{F} a_{s}=-0.8$ (blue dotted), -0.6 (green dashed), and -0.4 (red solid) for $P=P_{\text {edge }}$ in the case of $s=0.1$ and $E_{\mathrm{F}} / E_{\mathrm{R}}=2.5$. The swallowtail starts to appear at a critical value of $1 / k_{\mathrm{F}} a_{s} \approx-0.62$.

The blue dotted, green dashed, and red solid lines correspond to the cases where the interparticle interaction strengths are short, just enough, and sufficiently large for developing the swallowtails, respectively.

\section{Behavior of the Bloch Band near the value of the chemical potential with the change of the quasimomentum}

In Fig. 4(a) of the paper, the lowest three Bloch bands of the quasiparticle energy spectrum for $P=P_{\text {edge }}$ at $k_{\perp}=0$ and $1 / k_{\mathrm{F}} a_{s}=-0.62$ are given. To visualize the behavior of the second band (the red solid line in that figure) near the value of the chemical potential with the change of the quasimomentum $P$, we show the cases of $P / P_{\text {edge }}=0$ (black dotted), 0.5 (green dashed), and 1 (red solid) in the following Fig. 6. Notice that the sharp minima in the curves for $P / P_{\text {edge }}=0$ and -0.5 are due to avoided crossings with other bands.

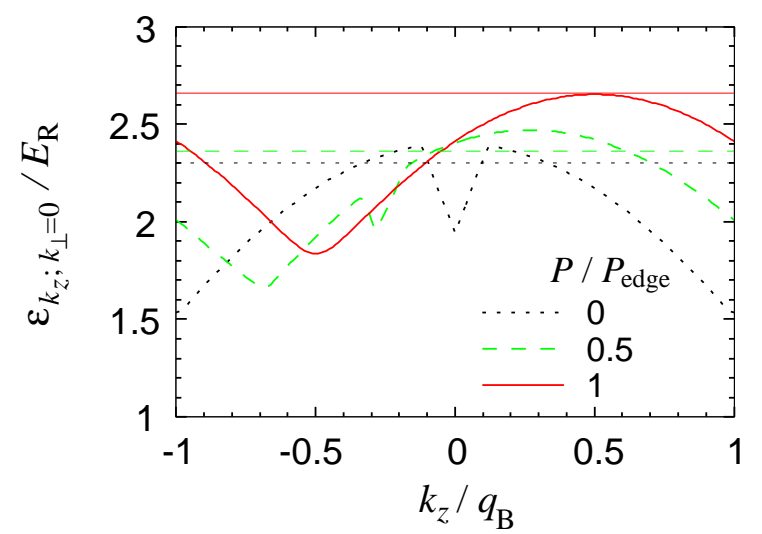

FIG. 6: Bloch band of the quasiparticle energy spectrum around the chemical potential for $P / P_{\text {edge }}=0$ (black dotted), 0.5 (green dashed), and 1 (red solid), respectively, at $k_{\perp}=0$ and $1 / k_{\mathrm{F}} a_{s}=-0.62$ in the case of $s=0.1$ and $E_{\mathrm{F}} / E_{\mathrm{R}}=2.5$. The each horizontal line denotes the value of the chemical potential for the corresponding value of $P$.

\section{Application of the swallowtail band structure to a Directed motion}

In Fig. 7, we show a schematic diagram for our proposal of the directed motion of an atomic wave packet. The basic idea is making use of the difference in the group velocity $v_{G}$ between the quadratic-like band with a swallowtail and the sinusoidal-like band. By modulating $1 / k_{\mathrm{F}} a_{s}$ in 


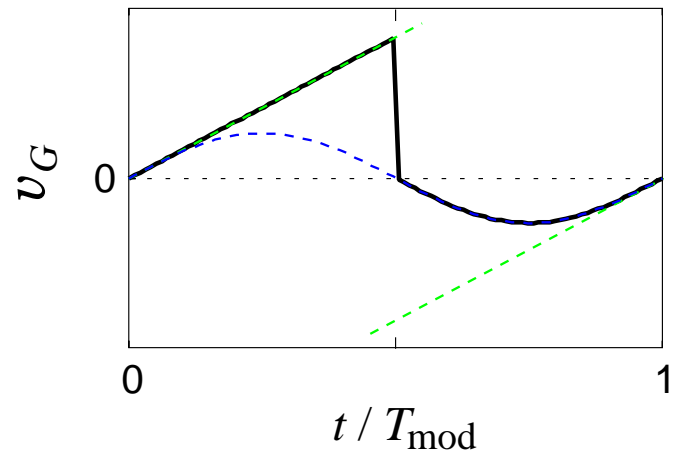

FIG. 7: Schematic diagram of the group velocity $v_{G}$ during one period $\left(T_{\mathrm{mod}}\right)$ of modulation of the magnetic field (accordingly, $\left.1 / k_{\mathrm{F}} a_{s}\right)$. The green (blue) dashed line shows the group velocity along the time when $1 / k_{\mathrm{F}} a_{s}$ is set to the value for a quadratic (sinusoidal) band structure as depicted in Fig. 1 (a) of the paper. When $1 / k_{\mathrm{F}} a_{s}$ is switched between the two values in a proper way, the group velocity of a wave packet will follow the black solid line. In real situation, the black line around $t=T_{\bmod } / 2$ is a smooth curve rather than a sharp drop due to a continuous variation of $1 / k_{\mathrm{F}} a_{s}$ connecting the two values. such a way that the dispersion is quadratic when $v_{G}$ is positive and sinusoidal when $v_{G}$ is negative, the net displacement over one period of modulation of $1 / k_{\mathrm{F}} a_{s}$ is positive and a directed motion can be produced. 